\title{
Solitary myofibroma of the mandible in an adult with magnetic resonance imaging and positron emission tomography findings: a case report
}

\author{
Yoko Tanaka ${ }^{1}$, Hiroyuki Yamada ${ }^{2 *}$, Tomoyuki Saito ${ }^{3}$, Kazutoshi Nakaoka ${ }^{2}$, Kenichi Kumagai ${ }^{2}$, Hisako Fujihara ${ }^{2}$, \\ Kenji Mishima ${ }^{4}$ and Yoshiki Hamada ${ }^{2}$
}

\begin{abstract}
Myofibroma is a benign tumor composed of myoid spindle cells. The prevalence of myofibroma in the oral cavity is very low, with the mandible being the most common site. This report describes an adult case of myofibroma that arose on the mandible and includes magnetic resonance imaging (MRI) and positron emission tomography (PET) findings. On the MRI T1-weighted images, the tumor appeared with signal iso-intensity and was highly and heterogeneously enhanced with contrast material. On the T2-weighted images, it appeared with increased signal intensity. ${ }^{18}$ F-fluorodeoxyglucose (FDG)-PET imaging showed abnormal strong accumulation of FDG in the left mandibular region. The tumor was removed by marginal resection of the left mandible under general anesthesia. Histopathological findings revealed that the tumor stroma contained abundant thin-walled vessels. The postoperative course was uneventful, and we found no evidence of recurrence at the postoperative 34-month follow-up.
\end{abstract}

Keywords: Myofibroma, Mandible, Adult, MRI, PET

\section{Background}

Myofibroma is a benign tumor composed of myoid spindle cells arranged around thin-walled blood vessels. Myofibromas are classified as either a solitary or multicentric type [1]. The solitary type is called myofibroma, whereas the multicentric type is known as myofibromatosis. The majority of myofibromas present in children less than 2 years of age [2]. Approximately half of myofibromas occur in the cutaneous and subcutaneous tissues of the head and neck region, followed by the trunk and the extremities. The other half occur in skeletal muscle or aponeuroses [1]. The prevalence of myofibroma in the oral cavity is very low, and the mandible is recognized as the most common site followed by the tongue and buccal mucosa [3]. Therefore, there are few reports of adult cases of oral myofibroma [4].

\footnotetext{
*Correspondence: yamada-hi@tsurumi-u.ac.jp

${ }^{2}$ Department of Oral and Maxillofacial Surgery, Tsurumi University School of Dental Medicine, 2-1-3 Tsurumi, Tsurumi-ku, Yokohama, Japan

Full list of author information is available at the end of the article
}

Some cases of oral myofibroma have been misdiagnosed as various benign and malignant tumors [5-7] because oral pathologists are infrequently exposed to soft tissue spindle cell neoplasms coupled with overlapping histologic patterns [5]. Although many reports dealing with oral myofibroma limited their focus to its pathological features [8,9], reports describing preoperative diagnostic imaging findings are few [10]. A preoperative image-based diagnosis using magnetic resonance imaging (MRI) and positron emission tomography (PET) would be helpful to determine whether the tumor is benign or malignant and to determine the treatment plan. In this paper, we report an adult case of myofibroma that arose on the mandible and emphasize the diagnostic value of preoperative MRI and PET findings.

\section{Case presentation}

A 52-year-old woman was referred to the Department of Oral and Maxillofacial Surgery, Tsurumi University Dental Hospital (Yokohama, Japan) on 30 October 2010 for diagnosis and treatment of a mass on the left mandible. The patient had noticed the mass for 1 month. The 
mass grew rapidly from the extraction socket of the lower mandibular second molar. Past medical history revealed hypertension and hyperlipidemia, which were adequately controlled with medication. The patient's family history was unremarkable. Physical examination revealed a rubbery sessile mass measuring $20 \times 15 \mathrm{~mm}$ on the left mandible (Figure 1). The overlying oral mucosa looked absent. There was neither neuroparalysis nor cervical lymphadenopathy.

MRI examination was performed with a 1.5 Tesla system (Intera $1.5 \mathrm{~T}$; Philips Healthcare, Eindhoven, The Netherlands). On the T1-weighted axial images, the mass appeared isointense and was highly and heterogeneously enhanced with gadolinium in the left mandibular region. On the T2-weighted axial images, it appeared with increased signal intensity. On short-tau inversion recovery coronal imaging, the mass also showed high signal intensity (Figure 2). The CT images (Brilliance CT 16; Philips Healthcare, Eindhoven, The Netherlands) showed no bony changes of the mandible (Figure 3 ). The PET study was performed with a dedicated PET scanner (ECAT ACCEL; Siemens Healthcare AG, Munich, Germany). Data were acquired after administration of $181.5 \mathrm{MBq}$ ${ }^{18}$ F-fluorodeoxyglucose (FDG), using the post-injection transmission acquisition method. The image showed abnormal strong accumulation of FDG in the left mandibular region with a maximum standard uptake value of 8.5. There were no abnormal accumulations of FDG in the cervical lymph nodes nor was there any uptake in the other organs (Figure 4A). The PET/CT fusion images showed that abnormal strong FDG accumulation corresponded to the mass on the left mandible (Figure 4B).

In November 2010, the biopsy was performed under local anesthesia. The pathological diagnosis was myofibroblastic tumor, probably benign. However, the mass enlarged rapidly to $35 \times 25 \mathrm{~mm}$ within 3 weeks after biopsy. According to the clinical findings, we could not completely deny the malignancy of the tumor. On 2 December 2010, it was removed by marginal resection of the left mandible under general anesthesia (Figure 5).

Pathological examination of hematoxylin and eosin (HE)-stained sections revealed that the tumor was composed of interlacing bundles of spindle-shaped cells. The tumor cells had tapered blunt-ended nuclei with palestaining eosinophilic cytoplasm. The spindle-shaped cells did not show either cellular atypism or polymegethism. Mitoses were sometimes observed. The stroma contained abundant thin-walled vessels (Figure 6A,B). Immunohistochemical staining was performed using an avidin-biotin method. Immunoreactivity for vimentin (mouse anti-vimentin monoclonal antibody, clone V9; DakoCytomation, Glostrup, Denmark), muscle actin (mouse anti-human muscle actin monoclonal antibody, clone HHF35; DakoCytomation), and $\alpha$-smooth muscle actin (mouse anti-human alpha smooth muscle actin monoclonal antibody, clone 1A4; Daco, Carpinteria, CA, USA) were positive in the tumor cells. Conversely, S-100 protein (rabbit anti-human S-100 polyclonal antibody, clone MOC32; Nichirei, Tokyo, Japan), desmin (mouse anti-human desmin monoclonal antibody, clone D33; Daco, Kyoto, Japan), and CD34 (mouse anti-human CD34 monoclonal antibody, clone NU-4A1; Nichirei, Tokyo, Japan) were negative. Thin-walled vessels were positive for CD34 (Figure 6C,D,E). These findings were consistent with the diagnosis of myofibroma.

The postoperative course was uneventful, and we found no evidence of recurrence at the postoperative 34month follow-up.

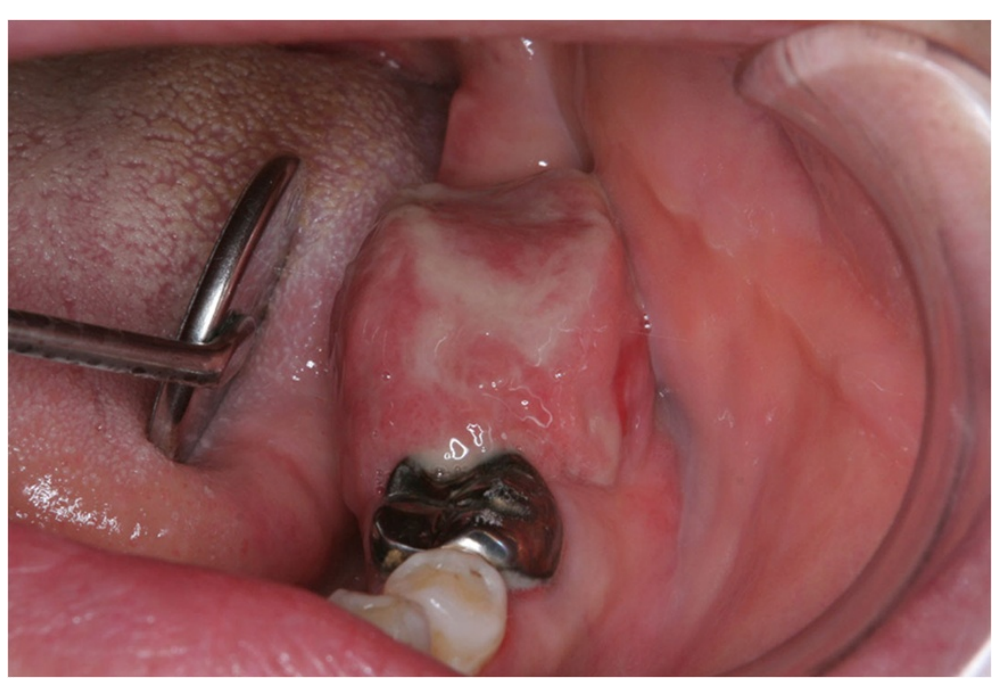

Figure 1 Photograph showing the mass on the mandible. 

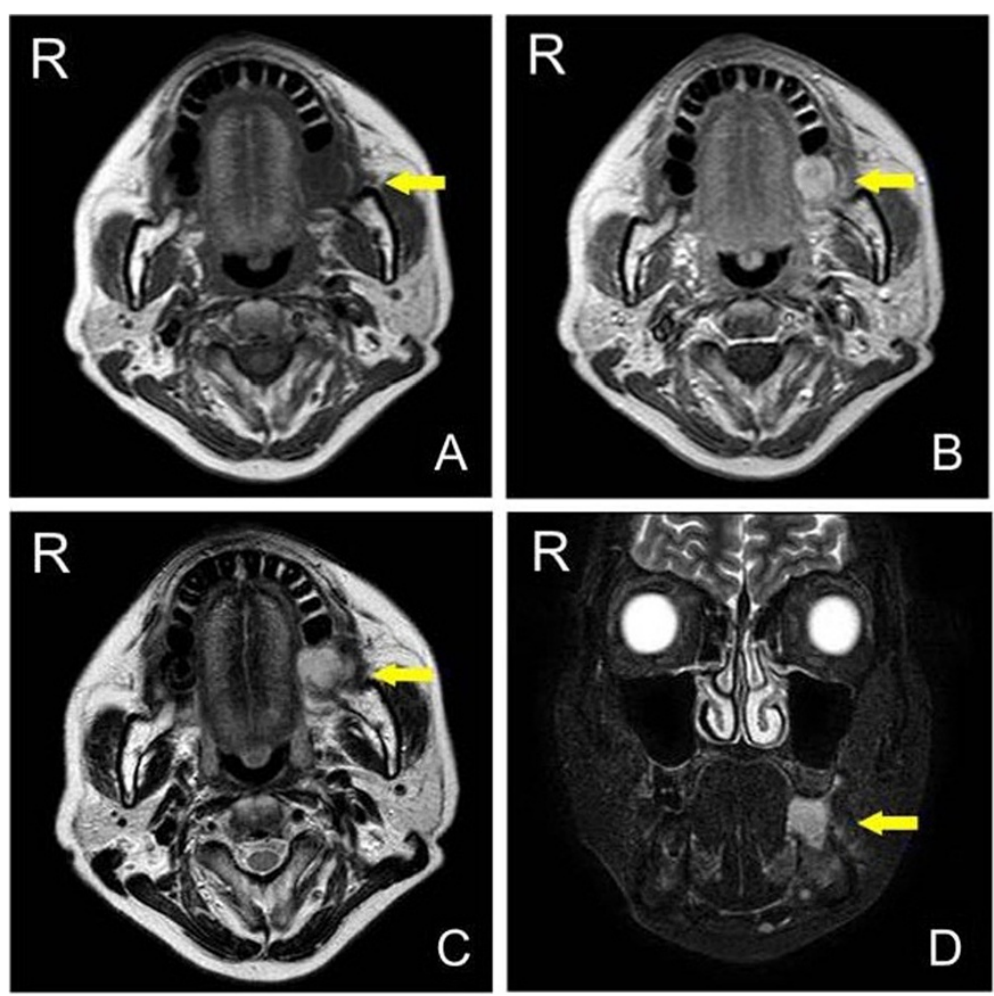

Figure 2 Preoperative MRI images. (A) T1-weighted axial image showing a mass of iso-intensity on the mandible (arrow). (B) Gadoliniumenhanced T1-weighted axial image showing a highly and heterogeneously enhanced mass (arrow). (C) T2-weighted axial image showing high signal intensity within the mass (arrow). (D) Short-tau inversion recovery images showing high signal intensity in the mass (arrow). MRI, magnetic resonance imaging.

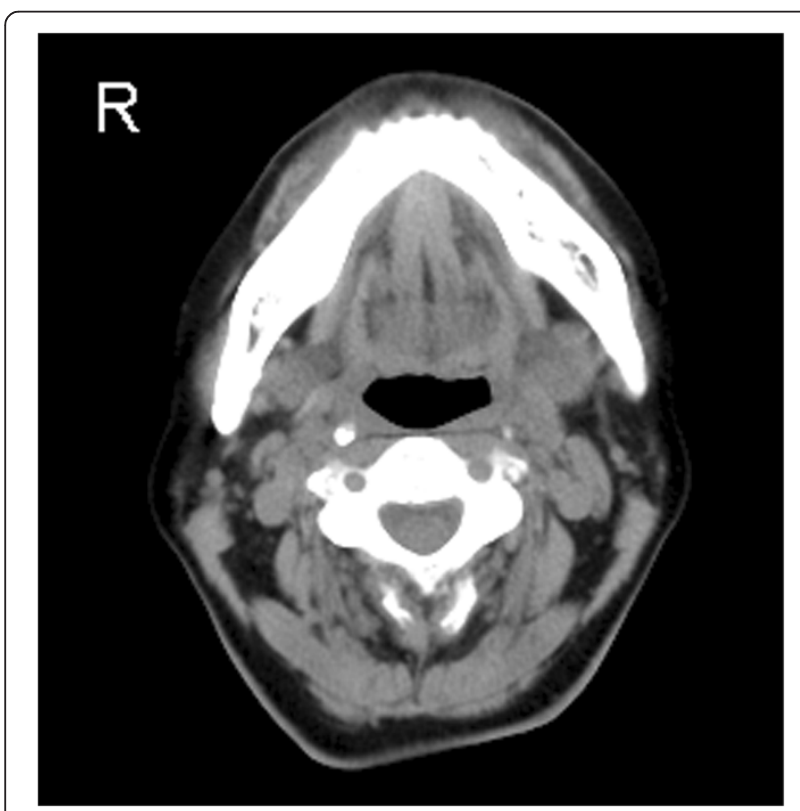

Figure $3 \mathrm{CT}$ image showing no bony changes of the mandible. $C T$, computed tomography.

\section{Discussion}

There are few reports of adult patients with myofibroma of the mandible [4]. Clinical differences between adults and pediatric patients with myofibroma have suggested that pediatric patients are likely to demonstrate bone involvement $[7,11]$ and that lesions in adults almost invariably presented as the solitary form, with exceptional cases of the multicentric form [6]. However, no specific differences in histologic features between adult and pediatric patients have been reported $[6,11]$. The clinical features of our solitary case without bone destruction are in accordance with those of adult oral myofibroma.

In the present case, the preoperative diagnosis based on the biopsy specimen findings was a myofibroblastic tumor, probably benign. However, the tumor grew rapidly in a few weeks. In the oral and maxillofacial region, approximately one-fourth of cases of myofibroma exhibit rapid enlargement, and some lesions show accelerated growth after incisional biopsy [11]. Spindle cell tumors are difficult to diagnose with a small piece of biopsy specimen alone [5,7]. Therefore, the final pathological diagnosis must rely on a specimen from the entire surgically removed lesion. Consequently, we performed marginal 

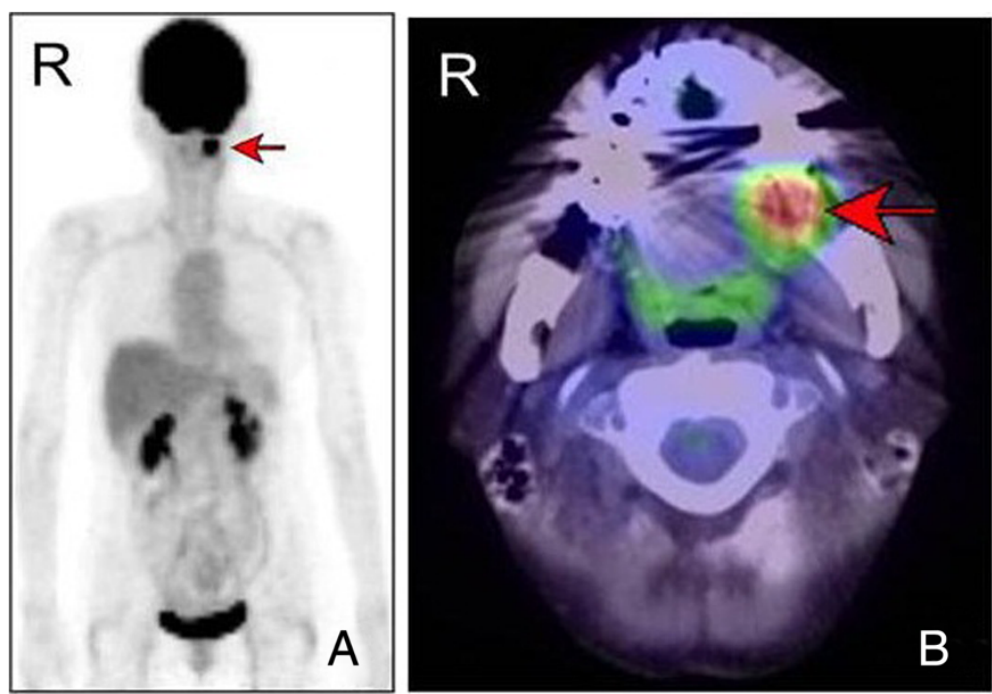

Figure 4 FDG-PET and PET/CT images. (A) FDG-PET coronal image of the mass. There is an abnormal accumulation of FDG in the left mandibular region (arrow). (B) PET/CT fusion image showing abnormal strong FDG accumulation corresponding to the mass on the left mandible (arrow). $\mathrm{CT}$, computed tomography; FDG, ${ }^{18} \mathrm{~F}$-fluorodeoxyglucose; PET, positron emission tomography.

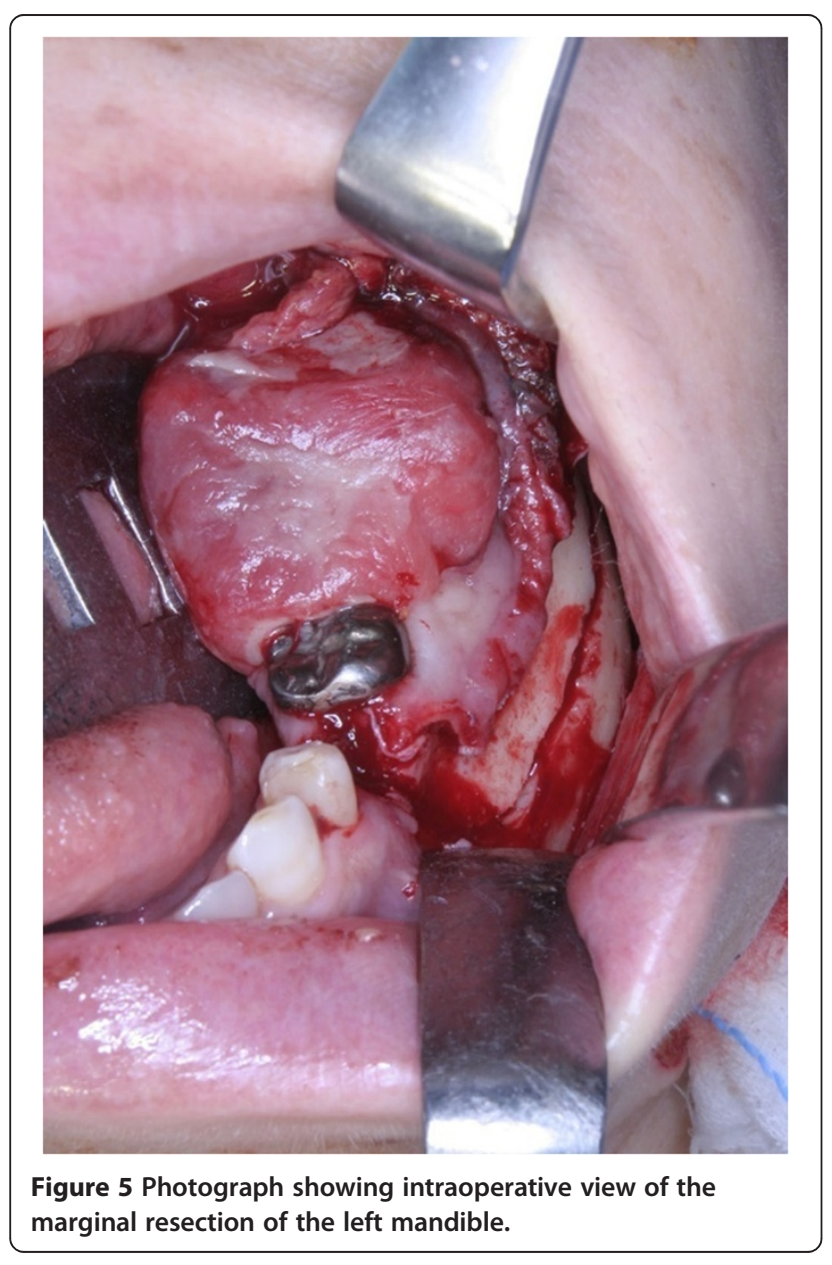

resection of the mandible with consideration of the possibility that the tumor was malignant.

To the best of our knowledge, PET findings of myofibroma have been reported in only one case in the literature. In that case, strong accumulation of FDG in a myofibroma of the mandible was documented [10]. The FDG-PET findings in the present case are in agreement with those of the previously reported case [10]. FDG accumulation could be caused partially by blood supply. For glucose to be taken up and used by tumor cells, an adequate blood supply is needed; therefore, FDG-PET is assessed by not only the glucose metabolism, but also the blood supply [12]. In our case, the strong FDG accumulation was assumed to be caused by activated glucose metabolism due to the high proliferative activity of tumor cells and rich blood supply to the tumor. High proliferative activity of tumor cells was proven macroscopically from the rapid growth of the tumor, and microscopically from the mitoses sometimes seen in the HE-stained sections. The rich blood supply to the tumor was evident from many blood vessels confirmed on the immunohistochemically stained sections, which showed CD34 positivity in vascular endothelial cells.

In the present case, gadolinium-enhanced T1-weighted MRI showed strong enhancement in the tumor. Strong enhancement on T1-weighted images by contrast material was also documented in some reported cases of myofibroma [13-15]. In the head and neck region, on the other hand, some tumors other than myofibroma (for example, hemangiomas, solitary fibrous tumors, carotid body tumors, and malignant tumors) also demonstrate strong contrast enhancement on T1-weighted images. However, 

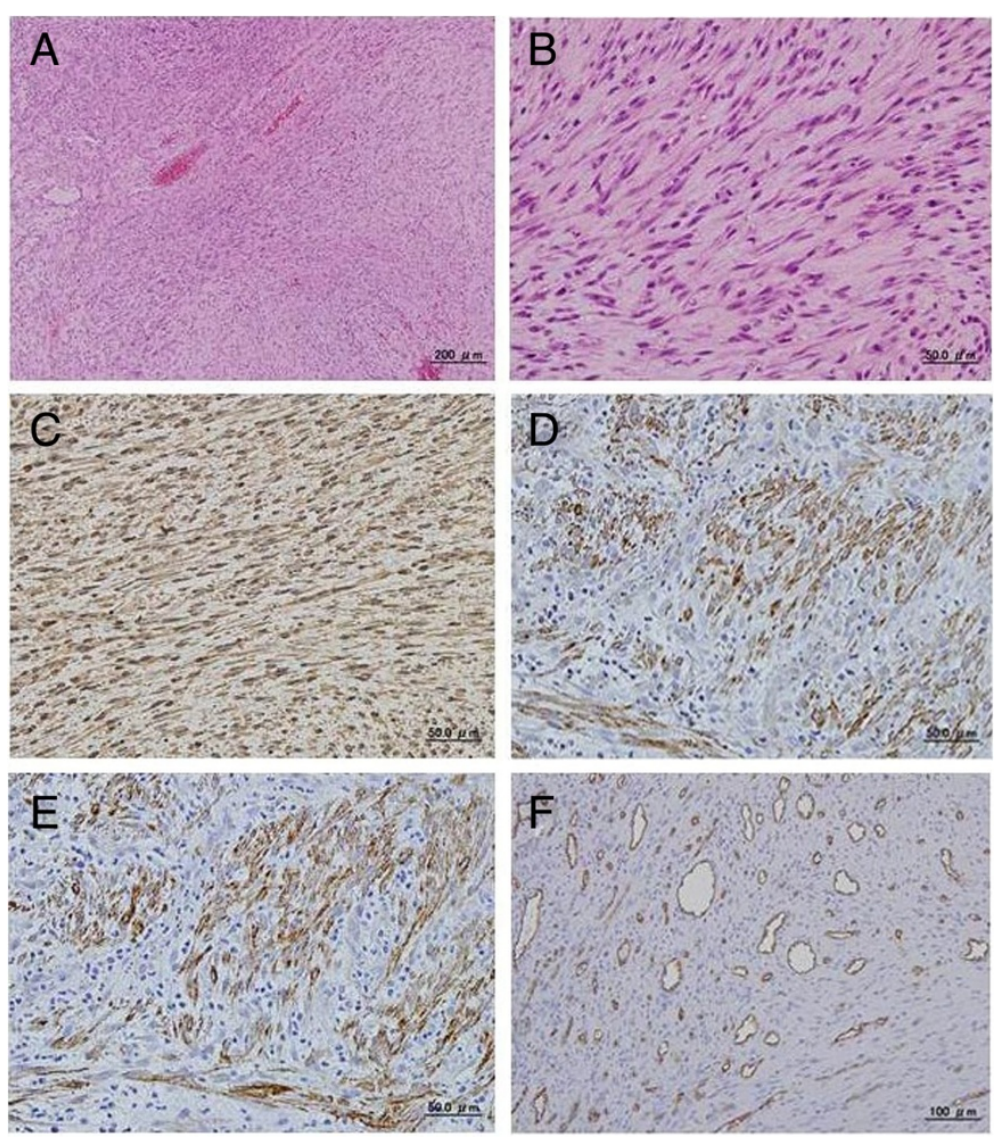

Figure 6 Photomicrographs showing spindle-shaped cells, cytoplasmic immunoreactivity and vascular endothelial cells. (A) Interlacing bundles of spindle-shaped cells (HE stain, original magnification $\times 100$ ). (B) Spindle-shaped cells with tapered blunt-ended nuclei and pale-staining eosinophilic cytoplasm (HE stain, original magnification $\times 400$ ). (C) Cytoplasmic positive immunoreactivity for vimentin (original magnification $\times 400$ ). (D) Cytoplasmic positive immunoreactivity for HHF-35 (original magnification $\times 400$ ). (E) Cytoplasmic positive immunoreactivity for a-SMA (original magnification $\times 400$ ). (F) Vascular endothelial cells with cytoplasmic positive immunoreactivity for CD34 (original magnification $\times 200$ ). $H E$, hematoxylin and eosin.

hemangioma [16] and solitary fibrous tumor [17] could be discriminated through FDG-PET findings of weak accumulation of FDG within the lesions. Moreover, carotid body tumors can be clinically ruled out by the specific site of the tumor [18]. However, malignant tumors are difficult to differentiate from myofibroma on the basis of MRI and PET findings. To avoid unnecessarily aggressive surgery, the clinician should be aware that myofibroma could show false positive results in a PET study aiming for detection of malignant tumors. Although a preoperative specific diagnosis of myofibroma could not be established in our case, clinical findings in combination with MRI and PET could narrow down the differential diagnoses. Complete local excision is performed for the treatment of solitary myofibroma [6]. Recurrence is rare, and such cases are usually controlled with re-excision [1,3]. In the present case, the tumor was completely removed by marginal resection of the left mandible and we found no evidence of recurrence at the postoperative 34-month follow-up.

\section{Conclusions}

In this report, we described an adult case of myofibroma that arose on the mandible and discussed the diagnostic value of preoperative MRI and PET findings.

\section{Consent}

Written informed consent was obtained from the patient for publication of this case report and any accompanying images. A copy of the written consent is available for review by the Editor-in-Chief of this journal.

\section{Abbreviations \\ CT: Computed tomography; FDG: ${ }^{18}$ F-fluorodeoxyglucose; HE: Hematoxylin and eosin; MRI: Magnetic resonance imaging; PET: Positron emission tomography.}

\section{Competing interests}

The authors declare that they have no competing interests.

\section{Authors' contributions}

YT and TS performed the operation. HF and TS were clinically responsible for the patient's care. KM was responsible for the pathology. KK and KN 
reviewed the literature. $Y T$ and $H Y$ wrote the main manuscript. $Y H$ revised the manuscript for important intellectual content. All authors read and approved the final manuscript.

\section{Author details}

'Department of Oral and Maxillofacial Surgery, Sendai Tokushukai Hospital, Sendai, Miyagi, Japan. ${ }^{2}$ Department of Oral and Maxillofacial Surgery, Tsurumi University School of Dental Medicine, 2-1-3 Tsurumi, Tsurumi-ku, Yokohama, Japan. ${ }^{3}$ Department of Oral and Maxillofacial Surgery, Nagano Matsushiro General Hospital, Matsushiro, Nagano, Japan. ${ }^{4}$ Department of Oral Pathology and Diagnosis, School of Dentistry, Showa University, Hatanodai,

Shinagawa-ku, Tokyo, Japan.

Received: 7 April 2013 Accepted: 15 March 2014

Published: 28 March 2014

\section{References}

1. Rubin B, Bridge J: Myofibroma/myofibromatosis. In Pathology and Genetics of Tumours of Soft Tissue and Bone. Edited by Fletcher CDM, Unni KK, Mertens F. Lyon: International Agency for Research on Cancer (IARC); 2002:59-61.

2. Chung EB, Enzinger FM: Infantile myofibromatosis. Cancer 1981, 48:1807-1818

3. Neville B, Damm D, Allen C, Bouquot J: Myofibroma (myofibromatosis). In Oral and Maxillofacial Pathology. 3rd edition. St Louis, MO: Saunders Elsevier; 2009:515-516.

4. Brierley DJ, Khurram SA, Speight PM: Solitary myofibroma of the adult mandible: a case report. Oral Surg Oral Med Oral Pathol Oral Radiol Endod 2013, 115:e40-e43.

5. Jordan RC, Regezi JA: Oral spindle cell neoplasms: a review of $\mathbf{3 0 7}$ cases. Oral Surg Oral Med Oral Pathol Oral Radiol Endod 2003, 95:717-724

6. Vered M, Allon I, Buchner A, Dayan D: Clinico-pathologic correlations of myofibroblastic tumors of the oral cavity: II myofibroma and myofibromatosis of the oral soft tissues. J Oral Pathol Med 2007, 36:304-314.

7. Allon I, Vered M, Buchner A, Dayan D: Central (intraosseous) myofibroma of the mandible: clinical, radiologic, and histopathologic features of a rare lesion. Oral Surg Oral Med Oral Pathol Oral Radiol Endod 2007, 103:e45-e53.

8. Azevedo Rde S, Pires FR, Della Coletta R, de Almeida OP, Kowalski LP, Lopes MA: Oral myofibromas: report of two cases and review of clinical and histopathologic differential diagnosis. Oral Surg Oral Med Oral Pathol Oral Radiol Endod 2008, 105:e35-e40.

9. Montgomery E, Speight PM, Fisher C: Myofibromas presenting in the oral cavity: a series of 9 cases. Oral Surg Oral Med Oral Pathol Oral Radiol Endod 2000, 89:343-348.

10. Shibuya Y, Takeuchi J, Sakaguchi H, Yokoo S, Umeda M, Komori T: Myofibroma of the mandible. Kobe J Med Sci 2008, 54:e169-e173.

11. Foss RD, Ellis GL: Myofibromas and myofibromatosis of the oral region: a clinicopathologic analysis of 79 cases. Oral Surg Oral Med Oral Pathol Oral Radiol Endod 2000, 89:57-65.

12. Schliephake $H$, Berding G, Knapp WH, Sewilam S: Monitoring of graft perfusion and osteoblast activity in revascularised fibula segments using $\left[{ }^{18} \mathrm{~F}\right]$-positron emission tomography. Int J Oral Maxillofac Surg 1999, 28:349-355

13. Konishi E, Mazaki T, Urata Y, Tanaka K, Kanoe H, Ikenaga M, Hayakawa K, Yanagisawa A: Solitary myofibroma of the lumbar vertebra: adult case. Skeletal Radiol 2007, 36(Suppl 1):S86-S90.

14. Rodrigues EB, Shields CL, Eagle RC Jr, Marr BP, Shields JA: Solitary intraosseous orbital myofibroma in four cases. Ophthal Plast Reconstr Surg 2006, 22:292-295

15. Kayes AV, Bancroft LW, Tennyson GS, O'Connor MI: Myofibroma of the upper arm in a 52-year-old woman. Skeletal Radiol 2002, 31:240-245.

16. Hatayama K, Watanabe H, Ahmed AR, Yanagawa T, Shinozaki T, Oriuchi N, Aoki J, Takeuchi K, Endo K, Takagishi K: Evaluation of hemangioma by positron emission tomography: role in a multimodality approach. J Comput Assist Tomogr 2003, 27:70-77.
17. Yamada H, Hamada Y, Fujihara H, Fukami K, Mishima K, Nakaoka K, Kumagai K, Imamura E: Solitary fibrous tumor of the buccal space resected in combination with coronoidectomy. Oral Surg Oral Med Oral Pathol Oral Radiol Endod 2012, 114:e9-e14.

18. Arya S, Rao V, Juvekar S, Dcruz AK: Carotid body tumors: objective criteria to predict the Shamblin group on MR imaging. AJNR Am J Neuroradiol 2008, 29:1349-1354

doi:10.1186/1477-7819-12-69

Cite this article as: Tanaka et al:: Solitary myofibroma of the mandible in an adult with magnetic resonance imaging and positron emission tomography findings: a case report. World Journal of Surgical Oncology 2014 12:69.

\section{Submit your next manuscript to BioMed Central and take full advantage of:}

- Convenient online submission

- Thorough peer review

- No space constraints or color figure charges

- Immediate publication on acceptance

- Inclusion in PubMed, CAS, Scopus and Google Scholar

- Research which is freely available for redistribution 